\title{
OCORRÊNCIA DE MYXOBOLUS SP. (MYXOZOA) EM THORACOCHARAX STELLATUS (KNER, 1858) (CHARACIFORMES) EM UM IGARAPÉ DA FLORESTA AMAZÔNICA, PARÁ, BRASIL
}

\author{
DE ARAÚJO, R.S. ${ }^{1}$; CORRÊA, F. ${ }^{2}$; DE SOUSA, F.B. ${ }^{3}$; RAMOS, A.B.M.A. ${ }^{3}$; NETO, J.L.S. ${ }^{3}$ \& MATOS, E.R. ${ }^{3}$ \\ 1. Universidade Federal do Acre, Laboratório de Ictiologia e Ecologia Aquática, Rio Branco, Acre, \\ Brasil. \\ 2. Instituto Federal do Amazonas - Campos Eirunepé, Amazonas (IFAM-Eirunepé), Eirunepé, Amazo- \\ nas, Brasil. \\ 3. Laboratório de Pesquisa Carlos Azevedo, Universidade Federal Rural da Amazônia, Belém, Pará, \\ Brasil. \\ *Corresponding author: rebecasilvade@gmail.com
}

\begin{abstract}
De Araújo, R.S. ; Corrêa, F.; de Sousa, F.B.; Ramos, A.B.M.A.; Neto, J.L.S. \& Matos, E.R., (2017). Ocorrência de Myxobolus sp. (MYXOZOA) EM Thoracocharax stellatus (KNER, 1858) (Characiformes) em um Igarapé da Floresta Amazônica, Pará, Brasil. Braz. J. Aquat. Sci. Technol. 18(2). elSSN 1983-9057. DOI: 10.14210/bjast.v21n1. Thoracocharax stellatus (KNER, 1858), belonging to the Gasteropelecidae family, is represented by small fish. The fish were captured using dragnets in an area adjacent to the Guamá River, with an average total length of $4.2 \pm 0.8 \mathrm{~cm}$ and total average weight of $0.9 \pm 0.3 \mathrm{~g}$. Fragments of gills were collected and analyzed fresh under a light microscope, where the presence of cysts full of spores of Myxobolus sp. with unequal polar capsules was observed. This study reports the first record of Myxobolus sp. found in T. stellatus coming from a tropical river in the State of Amazonia, in which the morphological characteristics differ from those of the other species when compared.

Key Words: Gasteropelecidae, Freshwater, Ornamental fish, Microparasites, Amazonia.
\end{abstract}

A família Gasteropelecidae é um grupo de peixes de pequeno porte alcançando até $12 \mathrm{~cm}$ no comprimento total e inclui cerca de nove espécies distribuídas em três gêneros: Carnegiella Eigenmann 1909; Gasteropelecus Bloch, 1784 e Thoracocharax Fowler, 1906, com distribuição em quase todos os países da região Neotropical, com exceção do Chile (Silva et al., 2009). Dentre os gêneros citados Thoracocharax apresenta duas espécies $T$. securis e $T$. stellatus, onde a segunda espécie possui hábito alimentar insetívoro (Netto-Ferreira et al., 2007). A espécie T. stellatus possui grande importância econômica, pois é uma das 725 espécies de peixes nativas permitidas para explotação com finalidade ornamental ou de aquariofilia em ecossistemas aquáticos da Amazônia (MPA, 2012). Entretanto, devido a essa importância o conhecimento sobre a fauna parasitária desta espécie é de fundamental relevância para a aquariofilia e ainda pouco se conhece sobre o assunto.

O filo Myxozoa Bütschli, 1882 agrupa aproximadamente 60 gêneros e mais de 2000 mil espécies descritas, com ampla distribuição em ambientes marinhos e de água doce (Casal et al., 2006; Lom \& Dykovà, 2006). Essas espécies são consideradas agentes patogênicos comumente encontrados em peixes de diferentes regiões do mundo, porém, já registradas em outros grupos taxonômicos como moluscos, anfíbios, répteis, pássaros e mamíferos (Feist \& Longshaw, 2006; Carriero et al., 2013). O gênero Myxobolus Bütschli, 1882, apresenta cerca de 785 espécies válidas, sendo um dos maiores grupos dos myxosporídeos (Lom \& Dykovà, 2006). Esses microparasitas possuem estágio de vida vegetativo, podendo ser encontrados nas cavidades corpóreas, órgãos vitais, tecidos intra e extracelulares, causando inflamações devido à formação de cistos, resultando em efeitos deletérios teciduais em seus hospedeiros (Kent et al., 2001; Azevedo et al., 2009; Azevedo et al., 2011). Nos últimos anos, várias espécies de Myxobolus tem sido descrita em diversas espécies de peixes que habitam ambientes marinhos, estuarinos e de águas continentais Eiras et al. (2005); Tajdari et al. (2005); Casal et al. (2006); Eiras et al. (2007); Azevedo et al. (2010); Milanin et al. (2010); Azevedo et al. (2011); Maciel et al. (2011); Velasco et al. (2012) e Carriero et al. (2013). Entretanto, devido a alta diversidade de peixes da região Neotropical, ainda existem inúmeras lacunas do conhecimento sobre a ocorrência deste parasito em muitas espécies de peixes, com isso o estudo reporta o primeiro registro de Myxobolus sp., 
em Thoracocharax stellatus um peixe ornamental de importância no mercado mundial da aquariofilia.

Os peixes foram capturados entre setembro de 2014 a janeiro de 2015 que compreende os períodos de alta e baixa precipitação, em um igarapé localizado a margem direita do rio Guamá (012 $27^{\prime} \mathrm{S}, 48^{\circ} 26^{\prime} \mathrm{W}$ ), Belém, Pará, Brasil. Para a captura dos exemplares foi utilizado uma tarrafa com malha de $10 \mathrm{~mm}$ entre nós adjacentes, posteriormente acondicionados em sacos plásticos com aeração artificial, contendo aproximadamente $20 \mathrm{~L}$ de água do igarapé. Os peixes foram transportados vivos para o Laboratório de Pesquisa Carlos Azevedo da Universidade Federal Rural da Amazônia, onde foram mantidos em aquário areado de $36 \mathrm{~L}$, até que todos fossem examinados.

Os peixes foram anestesiados com Tricaína Metanolsulfonato (MS222, SIGMA), na concentração de $50 \mathrm{mg} / \mathrm{L}$, eutanasiados conforme o protocolo do CEUA-UFRA 013/2014, em seguida foram mesurados o comprimento total (CT, mm) e o peso total (PT, g). Concomitantemente às coletas dos peixes, foram estimados os seguintes parâmetros ambientais: temperatura água $\left({ }^{\circ} \mathrm{C}\right)$, oxigênio dissolvido (OD), potencial hidrogeniônico $(\mathrm{pH})$ utilizando um multiparâmetro marca YSI. A fim de verificar a existência dos parasitas, os peixes foram eviscerados e os órgãos observados em estereomicroscópio binocular e quando confirmada a presença de cistos e esporos de Myxobolus sp., foram documentados a fresco utilizando microscópio trinocular ZEISS PRIMO STAR com câmera fotográfica Canon A610/A620 $52 \mathrm{~mm}$. A classificação quanto ao formato do corpo dos esporos elipsoidal, ovoide ou arredondada, foi de acordo com (Lom \& Dykovà, 2006) e as medidas morfométricas, tamanho da cápsula polar, quantidade de valvas e o número de voltas dos filamentos polares, de acordo com (Matos et al., 2001).

Foram analisados um total de 15 indivíduos

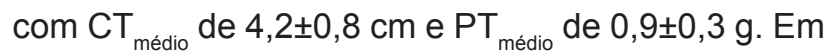
relação as variáveis ambientais a temperatura variou entre $32^{\circ} \mathrm{C}$ (setembro) a $23^{\circ} \mathrm{C}$ (janeiro), oxigênio dissolvido entre $7,1 \mathrm{mg} / \mathrm{l}$ (setembro) a $3,8 \mathrm{mg} / \mathrm{l}$ (janeiro) e $\mathrm{pH}$ de 6,1 (setembro) a 5,5 (janeiro). Dentre os exemplares analisados de $T$. stellatus, 10 apresentaram cistos de formato arredondado e esbranquiçados de Myxobolus sp., registrados entre os filamentos branquiais, sendo caracterizados por apresentarem corpo no formato piriforme, duas valvas e duas cápsulas polares desiguais (Figura 1). Em relação às medidas dos esporos o comprimento do corpo foi de $17 \mu \mathrm{m}$ e a largura foi de $8,23 \mu \mathrm{m}$, com duas cápsulas polares de tamanhos diferentes e formato oval, onde a maior capsula polar mediu 10,71 $\mu \mathrm{m}$ de comprimento e 4,47 $\mu \mathrm{m}$ de largura, apresentando 10-12 voltas de filamento polar e a menor capsula polar, mediu $4,59 \mu \mathrm{m}$ de comprimento e 1,7 $\mu \mathrm{m}$ de largura, apresentando 3-4 voltas de filamento polar (Figura 2).

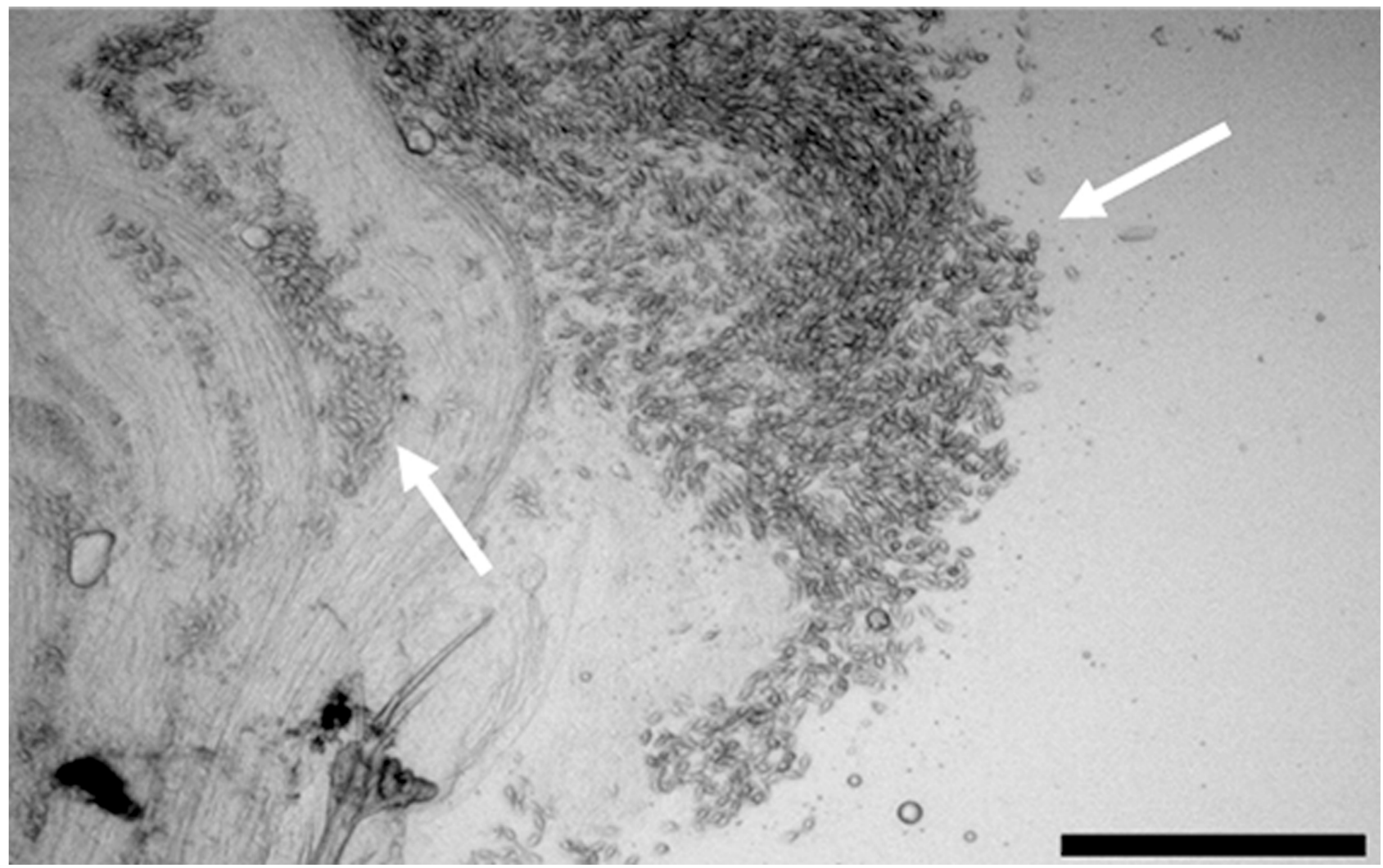

Figura 1 - Fotomicrografia de Myxobolus sp. 1 - Lâmina preparada com material a fresco mostrando cisto rompido liberando esporos maduros entre os filamentos branquiais de T. stellatus (seta). Barra $=200 \mu \mathrm{m}$. 


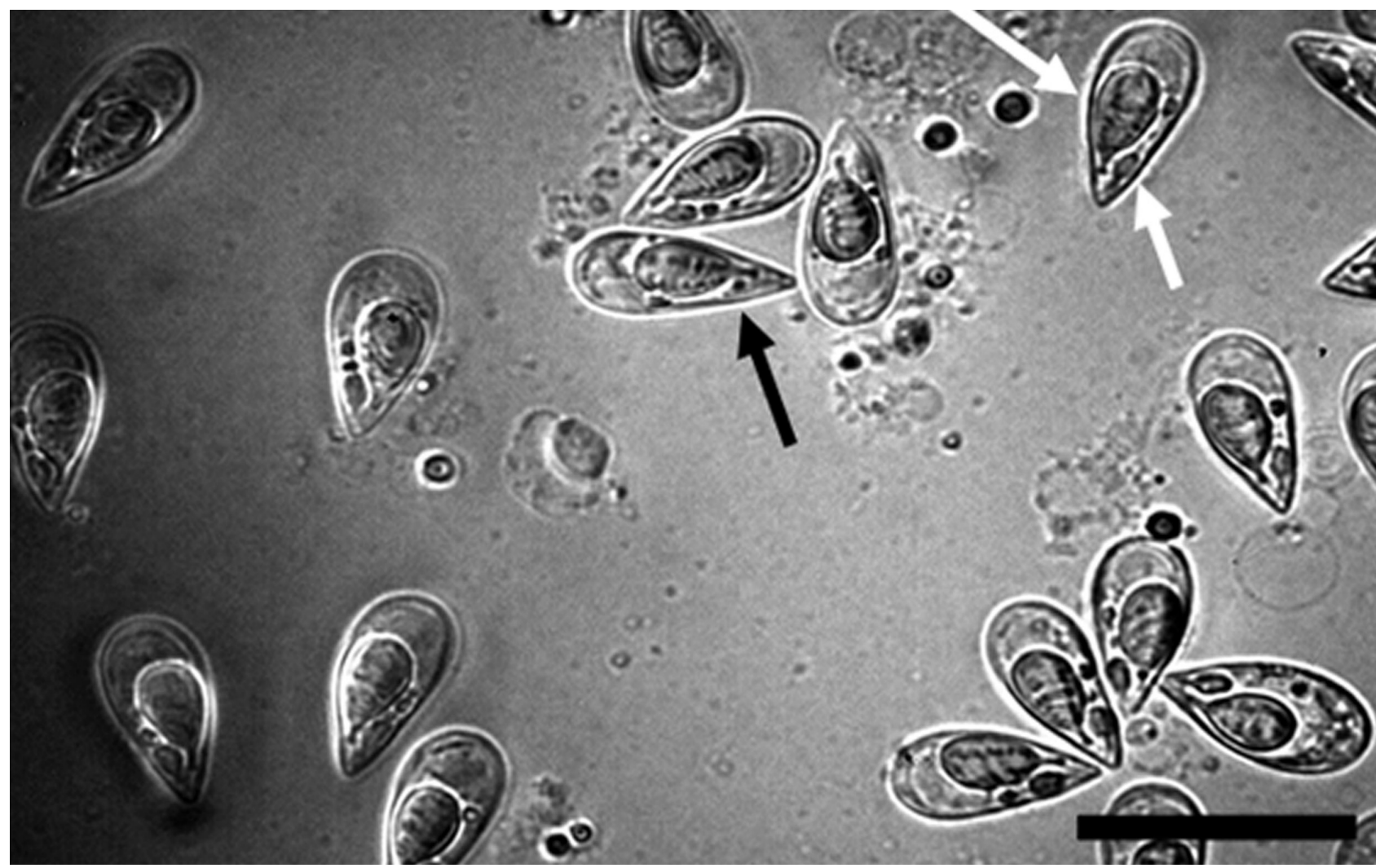

Figura 2 - Esporos maduros de Myxobolus sp. (seta preta) apresentando cápsulas polares desiguais (seta branca) livres nas brânquias de $T$. stellatus. Barra $=20 \mu \mathrm{m}$

A ocorrência deste gênero corrobora com outros autores, segundo Luque (2004) as espécies de parasitos que ocorrem com maior frequência em peixes marinhos e de água doce no Brasil são pertencentes ao gênero Myxobolus. São parasitos que formam cistos com numerosos esporos e podem provocar compressão nos tecidos ou órgãos do hospedeiro, onde Matos et al. (2001) destacam que estes são encontrados em órgãos importantes podendo ser letais para o hospedeiro, pois causam processos infecciosos agravando o quadro para lise celular. Um exemplo clássico na literatura são os danos causados por M. cerebralis, como a "doença do rodopio" ou a "doença da cauda negra", onde provocam distúrbios natatórios característicos em forma de círculos e quando localizados na cartilagem da coluna vertebral, exercem pressão sobre os nervos caudais deixando-a enegrecida (Eiras, 1994).

As características morfológicas e morfométricas de Myxobolus sp., do presente estudo é apresentada na tabela 1 comparado com outras espécies de Myxobolus descritas no Brasil, com destaque para a ocorrência de $M$. desaequalis parasitando branquiais de Apteronotus albifrons (Azevedo et al., 2002); M. metynnis encontrado em Metynnis argenteus parasitando tecidos subcutâneos (Casal et al., 2006); M. heckelii na brânquia do Centromochlus heckelii (Azevedo et al., 2009b); Myxobolus sp., no músculo cardíaco de Pimelodus ornatus (Matos et al., 2014) e M. Iomi nas brânquias do Prochilodus lineatus (Azevedo et al., 2014). Neste trabalho, foi encontrado cistos com esporos maduros de Myxobolus sp. e cápsulas polares de tamanhos diferentes parasitando filamentos branquiais de $T$. stellatus, corroborando com os trabalhos de Azevedo et al. (2002) que registrou para Apteronotus albifrons coletado no Estado do Pará a espécie de $M$. desaequalis e para Prochilodus lineatus, capturados no Estado de São Paulo a espécie M. Iomi (Azevedo et al., 2014), ambos parasitando as brânquias e apresentando cápsulas polares de tamanhos desiguais. Quanto ao sítio de infecção apresentou similaridade com o trabalho de Azevedo et al., (2009). Com relação ao tamanho dos esporos, tamanho das cápsulas polares e números de voltas dos filamentos polares, Myxobolus sp. do presente estudo diferenciou-se de todos os outros trabalhos apresentados. Diante do registro de Myxobolus sp. em T. stellatus e das características apresentadas pelo parasito, a continuidade do trabalho abordando aspectos moleculares é de fundamental importância para a determinação e classificação da espécie, na Amazônia Oriental, além de estudos sobre alimentação, a qual pode detectar um possível hospedeiro intermediário. 
De Araújo, R.S., et al. (2018). Ocorrência de Myxobolus sp., em Thoracocharax stellatus.

Tabela 1 - Dados morfométricos $(\mu \mathrm{m})$ de esporos maduros de Myxobolus sp., encontrado em T. stellatus e comparação com 263 outras espécies de Myxobolus descritas parasitando outras espécies de peixes no Brasil.

\begin{tabular}{|c|c|c|c|c|c|c|c|}
\hline Parasito & CT & LC & $\mathbf{C C P}$ & LCP & NFP & Sítio de infecção & Hospedeiro \\
\hline \multirow{2}{*}{ Myxobolus sp.* } & \multirow{2}{*}{17} & \multirow{2}{*}{8,23} & 10,71 & 4,47 & $10-12$ & \multirow{2}{*}{ Filamentos branquiais } & \multirow{2}{*}{ T. stellatus } \\
\hline & & & 4,59 & 1,7 & $3-4$ & & \\
\hline \multirow{2}{*}{$\begin{array}{l}\text { M. desaequalis (Azevedo et al., } \\
\text { 2002) }\end{array}$} & \multirow{2}{*}{18,3} & \multirow{2}{*}{11,2} & 11,2 & 4,9 & $11-12$ & \multirow{2}{*}{ Brânquias } & \multirow{2}{*}{ Apteronotus albifrons } \\
\hline & & & 4,6 & 2,8 & $4-5$ & & \\
\hline M. metynnis (Casal et al., 2006) & 13,1 & 7,8 & 5,2 & 2,3 & $8-9$ & Tecidos subcutâneos & Metynnis argenteus \\
\hline $\begin{array}{l}\text { M. heckelii (Azevedo et al., } \\
2009 \text { b) }\end{array}$ & $12.2-13.2$ & $6.3-6.9$ & $2.7-3.1$ & $1.4-2.0$ & $4-5$ & Brânquias & Centromochlus heckelii \\
\hline \multirow[t]{2}{*}{ Myxobolus sp. (Matos et al., 2014) } & $8 \pm 0.2$ & $5.8 \pm 0.4$ & $3.6 \pm 0.3$ & $1.2 \pm 0.2$ & - & Músculo cardíaco & Pimelodus ornatus \\
\hline & & & $6.4 \pm 0.9$ & $3.1 \pm 0.7$ & $8-11$ & & \\
\hline M. lomi (Azevedo et al., 2014) & $14.2 \pm 1.4$ & $11.1 \pm 1.5$ & $6.0 \pm 0.8$ & $2.9 \pm 0.5$ & & Brânquias & Prochilodus lineatus \\
\hline
\end{tabular}

Nota: Comprimento total (CT), Largura do corpo (LC), Comprimento da cápsula polar (CCP), Largura da cápsula polar (LCP), Número de voltas do filamento polar (NFP). Presente estudo $\left(^{\star}\right)$.

\section{AGRADECIMENTOS}

Á CAPES, PVE 88881.064967/2014-01), CNPq UNIVERSAL/ 2014, N. 441645/2014-3, CNPq/ Produtividade (CNPq 300949/2012-0), FAPESPA, SISBIO/ICMBIO-IBAMA, por autorizar a licença de pesquisa no. 27119, ao Laboratório de Pesquisa Edilson Matos (LPEM-UFPA) e aos alunos de graduação e de pós-graduação, pelo auxílio na coleta em campo e análises no laboratório.

\section{REFERÊNCIAS}

AZEVEDO, C.; CORRAL, L.; MATOS, E. 2002. Myxobolus desaequalis n. sp. (Myxozoa, Myxosporea), parasite of the Amazonian freshwater fish, Apteronotus albifrons (Teleostei, Apteronotidae). J Eukaryot Microbiol. 49(6): 485-488.

AZEVEDO, C.; CASAL, G.; MATOS, P.; FERREIRA, I.; MATOS, E. 2009. Light and electron microscopy of the spore of Myxobolus heckelii n. sp. (Myxozoa), parasite from the Brazilian fish Centromochlus heckelii (Teleostei: Auchenipteridae). J Eukaryot Microbiol. 56(6): 589-593.

AZEVEDO, C.; CASAL, G.; MENDONÇA, I.; CARVALHO, E.; MATOS, P. and MATOS, E. 2010. Light and electron microscopy of Myxobolus sciades n. sp. (Myxozoa), a parasite of the gills of the Brazilian fish Sciades herzbergii (Block, 1794) (Teleostei: Ariidae). Mem. Inst. Oswaldo Cruz. 105(2): 203-207.

AZEVEDO, C.; CASAL, G.; MARQUES, D.; SILVA, E.; MATOS, E. 2011. Ultrastructure of Myxobolus brycon n. sp. (Phylum Myxozoa), parasite of the piraputanga fish Brycon hilarii (Teleostei) from Pantanal (Brazil). J Eukaryot Microbiol. 58(2): 88-93.

AZEVEDO, R. K.; VIEIRA, D. H. M. D.; VIEIRA, G. H.; SILVA, R. J.; MATOS, E.; ABDALLAH, V. D. 2014. Phylogeny, ultrastructure and histopathology of Myxobolus lomi sp. nov., a parasite of Prochilodus lineatus (Valenciennes, 1836) (Characiformes: Prochilodontidae) from the Peixes River, São Paulo State, Brazil. Parasitol Int. 63(2): 303-307.

CARRIERO, M. M.; ADRIANO, E. A.; SILVA, M. R. M.; CECCARELLI, P. S.; MAIA, A. A. M. 2013. Molecular phylogeny of the Myxobolus and Henneguya genera with several new south American species. PLoS ONE. 8(9): 1-12.

CASAL, G.; MATOS, E.; AZEVEDO, C. 2006. A new myxozoan parasite from the Amazonian fish Metynnis argenteus (Teleostei: Characidae): light and electron microscope observations. J Parasitol. 92(4): 817-821.

EIRAS, J. C. 1994. Elementos de Ictioparasitologia. $1^{\circ}$ Volume. Fundação Engenheiro António de Almeida. Porto, 339p.

EIRAS, J. C.; MOLNÁR, K.; LU, Y. S. 2005. Synopsis of the species of Myxobolus Bütschli, 1882 (Myxozoa: Myxosporea: Myxobolidae). Syst Parasitol. 61(1): $1-46$.

EIRAS, J. C.; ABREU, P. C.; ROBALDO, R.; PEREIRAJUNIOR, J. 2007. Myxobolus platanus n. sp. (Myxosporea, Myxobolidae), parasite of Mugil platanus Gu"nther, 1880 (Osteichthyes, Mugilidae) from Lagoa dos Patos, RS, Brazil. Arq Bras Med Vet Zootec. 59(4): 895-898.

FEIST, S. W.; LONGSHAW, M. 2006. Phylum Myxozoa. 
In: Woo, P.T.K, (ed.) Fish diseases and disorders: Protozoan and Metazoan infections. UK: CAB International 230-296pp.

KENT, M. L.; ANDREE, K. B.; BARTHOLOMEW. J. L.; EL-MATBOULI.; M.; DESSER, S. S. 2001. Recent advances in our knowledge of the Myxozoa. J Eukaryot Microbiol. 48(4): 395-413.

LOM, J.; DYKOVÁ, I. 2006. Myxozoan genera: definition and notes on taxonomy, life-cycle terminology and pathogenic species. Folia Parasitol. 53(1): 1-36.

LUQUE, J. L. 2004. Biologia, epidemiologia e controle de parasitos de peixes. Rev Bras Parasitol Vet. Ouro Preto, MG, v. 13. Suplemento 1.

MACIEL, P. O.; AFFONSO, E. G.; BOIJINK, C. L.; TAVARES-DIAS, M.; INOUE, L. A. K. A. 2011. Myxobolus sp. (Myxozoa) in the circulating blood of Colossoma macropomum (Osteichthyes, Characidae). Rev Bras Parasitol Vet. 20(1): 82-84.

MATOS, E.; CORRAL, L.; MATOS, P.; CASAL, G.; AZEVEDO, C. 2001. Incidência de parasitas do Philum Myxozoa (Sub-reino Protozoa) em peixes da região amazônica, com especial destaque para o gênero Henneguya. Rev Cienc Agr. 36: 83-99.

MATOS, E.; VIDEIRA, M.; VELASCO, M.; SANCHES, O. 2014. Infection of the heart of Pimelodus ornatus (Teleostei, Pimelodidae), by Myxobolus sp. (Myxozoa, Myxobolidae). Rev Bras Parasitol Vet. 23(4): 543-546.

MILANIN, T.; EIRAS, J.C.; ARANA.; S.; MAIA, A. A.; ALVES.; A. L. 2010. Phylogeny, ultrastructure, histopathology and prevalence of Myxobolus oliveirai sp. nov., a parasite of Brycon hilarii (Characidae) in the Pantanal wetland, Brazil. Mem Inst Oswaldo Cruz. 105(6): 762-769.

MPA. Instrução normative interministerial no 1 de 3 de janeiro de 2012. Brasília, DF, 46 p, 2012.

NETTO-FERREIRA, A. L.; ALBRECHT, M. P.; NESSIMIAN, J. L.; CARAMASCHI, E. P. 2007. Feeding habits of Thoracocharax stellatus (Characiformes: Gasteropelecidae) in the upper rio Tocantins, Brazil. Neotrop. Ichthyol. 5(1): 69-74.

SILVA, E. L.; CENTROFANTE, L.; MIYAZAWA, C. S. 2009. Análise morfométrica em Thoracocharax stellatus (Kner, 1858) (Characiformes, Gasteropelecidae) proveniente de diferentes bacias hidrográficas Sul-americanas. Biota Neotrop. 9(2): 71-76.

TAJDARI, J.; MATOS, E.; MENDONÇA, I.; AZEVEDO, C. 2005. Ultrastructural morphology of Myxobolus testicularis sp. n., parasite of the testis of Hemiodopsis microlepis (Teleostei: Hemiodontidae) from the NE of Brazil. Acta Protozool. 44: 377-384. 